\title{
Two-Layer Predictive Control of a Continuous Biodiesel Transesterification Reactor
}

\author{
Hongyan Shi, ${ }^{1,2}$ Dingding Wang, ${ }^{3}$ Decheng Yuan, ${ }^{2}$ and Tianran Wang ${ }^{1}$ \\ ${ }^{1}$ Shenyang Institute of Automation, Chinese Academy of Sciences, Shenyang 110016, China \\ ${ }^{2}$ College of Information Engineering, Shenyang University of Chemical Technology, Shenyang 110142, China \\ ${ }^{3}$ College of Information Engineering, Zhejiang University of Technology, Hangzhou 310023, China \\ Correspondence should be addressed to Hongyan Shi; shihongyan@sia.cn
}

Received 19 July 2013; Accepted 29 August 2013

Academic Editor: Baocang Ding

Copyright (C) 2013 Hongyan Shi et al. This is an open access article distributed under the Creative Commons Attribution License, which permits unrestricted use, distribution, and reproduction in any medium, provided the original work is properly cited.

\begin{abstract}
A novel two-layer predictive control scheme for a continuous biodiesel transesterification reactor is presented. Based on a validated mechanistic model, the least squares (LS) algorithm is used to identify the finite step response (FSR) process model adapted in the controller. The two-layer predictive control method achieves the steady-state optimal setpoints and resolves the multivariable dynamic control problems synchronously. Simulation results show that the two-layer predictive control strategy leads to a significant improvement of control performance in terms of the optimal set-points tracking and disturbances rejection, as compared to conventional PID controller within a multiloop framework.
\end{abstract}

\section{Introduction}

With the depletion of fossil fuels and global environmental degradation, the development of alternative fuels from renewable resources has received considerable attention. Biodiesel has become the foremost alternative fuel to those refined from petroleum products. It can be produced from renewable sources, such as vegetable and animal oils, as well as from wastes, such as used cooking oil. Transesterification is the primary method of converting these oils to biodiesel [1-3]. A block diagram for a biodiesel production process by transesterification is shown in Figure 1.

A modern transesterification plant is continuous instead of batch. A continuous biodiesel production leads to better heat economization, better product purity from phase separation by removing only the portion of the layer furthest from the interface, better recovery of excess methanol in order to save on methanol cost and regulatory issues, minimal operator interference in adjusting plant parameters, and lower capital costs per unit of biodiesel produced. The same technology can also be applied to other biofuels production [4$6]$.
Biodiesel transesterification reactor is the most crucial operation unit to be controlled because any drift in standard operating condition may lead to significant changes in process variable and production quality specification [47]. These reactors have complicated dynamics and heat transfer characteristics. Moreover, they are inherently concerned with nonlinearity which arises from fluctuations of reactant concentration, reactant temperature, coolant temperature, and instrumentation noise or complex microbial interactions. The complicated nonlinear, multivariable, and coupling in nature are the fundamental control problems involved in biodiesel reactor $[8,9]$.

Recently, a number of reports have appeared on the controller design and dynamic optimization in continuous and batch biodiesel reactors. Mjalli et al. developed a rigorous mechanistic model of a continuous biodiesel reactor and proposed a multimodel adaptive control strategy which realized the set-point tracking and disturbance rejection [4]. Ho et al. further adopted adaptive generalized predictive control strategy to handle multivariable problems of a biodiesel reactor [8]. Wali et al. proposed an artificial intelligence technique to design online genetic-ANFIS temperature control based 


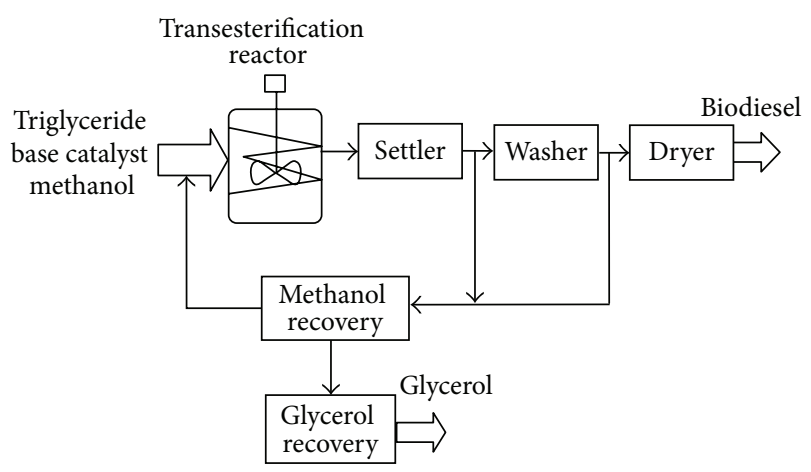

FIGURE 1: Biodiesel production by transesterification.

on LabVIEW for a novel continuous microwave biodiesel reactor [10]. Benavides and Diwekar realized the optimal control of a batch biodiesel reactor involved optimization of the concentration based on maximum principle [11].

This work considers the advanced control strategy of biodiesel continuous transesterification reactor. Model predictive control (MPC) is one of the most popular advanced control strategies. It is a class of model-based control algorithm, which has become a complex standard process industry solving complicated constrained multivariable control problems, and widely used in the chemical and petrochemical processes [12]. The main technical characteristics of MPC, include using mathematical models and history input and output data to predict future output, combined with the established control objectives, to calculate the optimal feedback rate. Compared with the traditional multiloop PID controllers, MPC takes into account simultaneously the effects of all manipulated variables to all controlled variables. Usually successfully put into operation, MPC can significantly reduce the standard deviation of the controlled variable and then through the card edge operations, improve the overall efficiency of the control system.

In recent years, there has been an integrated steadystate optimization of the two-layer predictive control strategy in MPC industry technology [13-15]. Two-layer predictive control is divided into upper steady-state optimization (SSO) layer and lower dynamic control layer. SSO can achieve real time optimization (RTO) objectives tracking asymptotically, independently complete local economic optimization of the corresponding MPC procedure. Specifically, the upper SSO uses steady-state gain of MPC dynamic mathematical model as the mathematical model and searches the optimum value within the constraints space of MPC. Part steady-state values of the operating or output variables will be in the position of "card edge". The calculation results of the SSO layer will be as the set- points to the lower MPC layer.

Although two-layer predictive control strategy has been widely used in many applications of chemical reactors, hardly any work was done on the biodiesel transesterification reactor. In this paper, a two-layer predictive control strategy is designed, tested, and simulated on a continuous biodiesel transesterification reactor. The scheme can amplify the advantages of both technologies in terms of process stability, and optimal and improved performances. Section 2 discusses the transesterification mechanism, which uses a validated mechanistic model of Mjalli et al. [4]. Then the twolayer predictive control strategy is developed in Section 3. Section 4 gives the control system design based on twolayer predictive control theory. Section 5 discusses model identification results and the performances of the control strategy.

\section{Mathematical Models}

The modeling of transesterification reactors starts with understanding the complex reaction kinetic mechanism. The stoichiometry of vegetable oil methanolysis reaction requires three mol of methanol (A) and one mol of triglyceride (TG) to give three mol of fatty acid methyl ester (E) and one mol of glycerol $(G)[16]$. The overall reaction scheme for this reaction is

$$
\mathrm{TG}+3 \mathrm{~A} \longleftrightarrow 3 \mathrm{E}+\mathrm{G} .
$$

The methanolysis, in turn, consists of three consecutive reversible reactions, where a mole of fatty acid methyl ester is released in each step, and monoglycerides (MG) and diglycerides (DG) are intermediate products. The stepwise reactions are

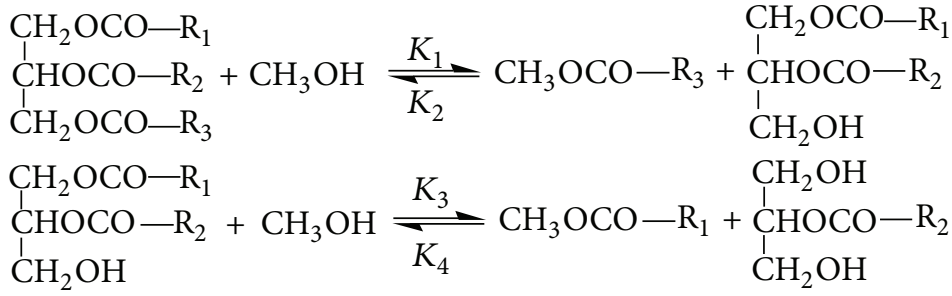

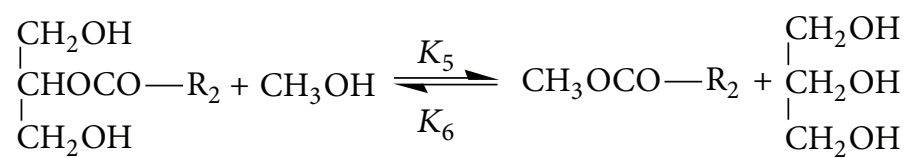


The stepwise reactions can be termed as pseudo-homogeneous catalyzed reactions, following second-order kinetics. The second-order kinetic model can be explained through the following set of differential equations [17]:

$$
\begin{gathered}
\frac{d C_{\mathrm{TG}}}{d t}=-k_{1}^{\prime} C_{\mathrm{TG}} C_{\mathrm{A}}+k_{2}^{\prime} C_{\mathrm{DG}} C_{\mathrm{E}}, \\
\frac{d C_{\mathrm{DG}}}{d t}=k_{1}^{\prime} C_{\mathrm{TG}} C_{\mathrm{A}}-k_{2}^{\prime} C_{\mathrm{DG}} C_{\mathrm{E}}-k_{3}^{\prime} C_{\mathrm{DG}} C_{\mathrm{A}}+k_{4}^{\prime} C_{\mathrm{MG}} C_{\mathrm{E}}, \\
\frac{d C_{\mathrm{MG}}}{d t}=k_{3}^{\prime} C_{\mathrm{DG}} C_{\mathrm{A}}-k_{4}^{\prime} C_{\mathrm{MG}} C_{\mathrm{E}}-k_{5}^{\prime} C_{\mathrm{MG}} C_{\mathrm{A}}+k_{6}^{\prime} C_{\mathrm{GL}} C_{\mathrm{E}}, \\
\frac{d C_{\mathrm{E}}}{d t}=k_{1}^{\prime} C_{\mathrm{TG}} C_{\mathrm{A}}-k_{2}^{\prime} C_{\mathrm{DG}} C_{\mathrm{E}}+k_{3}^{\prime} C_{\mathrm{DG}} C_{\mathrm{A}}-k_{4}^{\prime} C_{\mathrm{MG}} C_{\mathrm{E}} \\
+k_{5}^{\prime} C_{\mathrm{MG}} C_{\mathrm{A}}-k_{6}^{\prime} C_{\mathrm{GL}} C_{\mathrm{E}}, \\
\frac{d C_{\mathrm{A}}}{d t}=-\frac{d C_{\mathrm{E}}}{d t}, \\
\frac{d C_{\mathrm{GL}}}{d t}=k_{5}^{\prime} C_{\mathrm{MG}} C_{\mathrm{A}}-k_{6}^{\prime} C_{\mathrm{GL}} C_{\mathrm{E}},
\end{gathered}
$$

where $C_{\mathrm{TG}}, C_{\mathrm{DG}}, C_{\mathrm{MG}}, C_{\mathrm{E}}, C_{\mathrm{A}}$, and $C_{\mathrm{GL}}$ are concentrations of triglyceride, diglyceride, monoglyceride, methyl ester, methanol, and glycerol, respectively. $k_{1}^{\prime}, k_{3}^{\prime}$, and $k_{5}^{\prime}$ are the effective rate constants for the forward reactions, and $k_{2}^{\prime}, k_{4}^{\prime}$, and $k_{6}^{\prime}$ are the effective rate constants for the reverse reactions.

The previously selected kinetic model can be formulated in terms of a general reaction equation

$$
r_{j}=k_{j}^{\prime}\left[C_{i}\right]^{2} .
$$

The catalyst concentration remained constant because the sidereactions that consume the catalyst were supposed to be negligible. Therefore, each effective rate constant includes the catalyst concentration $\left(C_{\text {cat }}\right)$ and the corresponding rate constant for the catalyzed reaction [18]:

$$
k_{j}^{\prime}=k_{j} C_{\text {cat }} .
$$

The temperature influence on the reaction rate was studied from the Arrhenius equation (6) that shows the temperature dependency of the reaction rate constant

$$
k_{j}=k_{0} e^{\left(-E_{a} / R T\right)},
$$

where $k_{0}$ is a constant called the preexponential factor, $E_{a}$ is the activation energy of the reaction, and $R$ is the gas constant.

In order to realize the optimization and control of continuous biodiesel production process, the model used in the paper on the basis of the second-order kinetic model jointing the material and energy balance equations as well as the dynamic equation of the coolant temperature. The material balance for each component is expressed as follows [4]:

$$
V \frac{d C_{i}}{d t}=F_{i 0} C_{i 0}-F_{i} C_{i}-\sum_{j=1}^{n} r_{j} V
$$

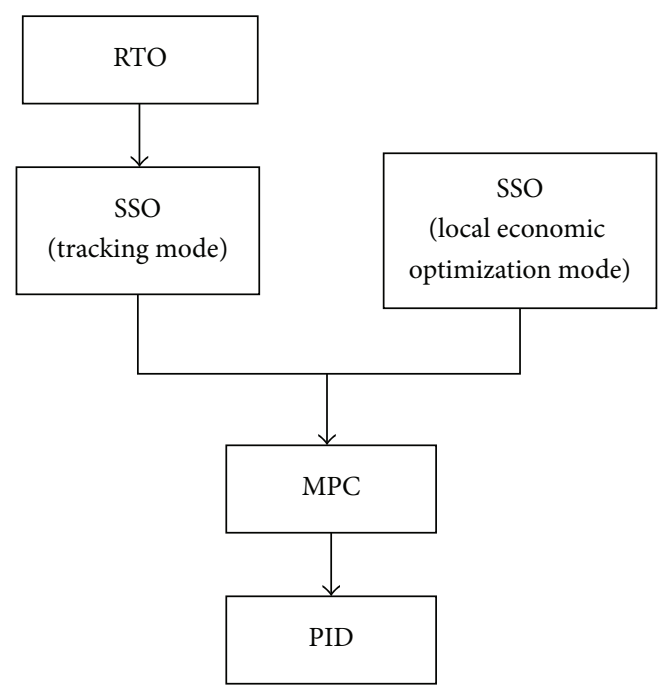

FIGURE 2: Framework of two-layer predictive control of industrial processes.

The reactor energy balance is expressed as

$$
\begin{aligned}
V \sum_{i=1}^{n_{i}} C_{i} C_{p_{i}} \frac{d T}{d t}= & \left(F_{\mathrm{TG} 0} C_{\mathrm{TG} 0} C_{P_{\mathrm{TG}}}+F_{\mathrm{A} 0} C_{\mathrm{A} 0} C_{P_{\mathrm{A}}}\right)\left(T_{0}-T\right) \\
& -\left(V \sum_{i=j}^{n} r_{j} \Delta H_{j}\right)-\left(U A_{H} \Delta T\right) .
\end{aligned}
$$

The coolant fluid energy balance is expressed as

$$
\frac{d T_{C}}{d t}=\frac{F_{C_{0}}}{V_{C}}\left(T_{C_{0}}-T_{C}\right)+\frac{U A_{H} \Delta T}{\rho_{C} V_{C} C_{P_{C}}} .
$$

The function equation of heat transfer coefficient is approximately expressed as

$$
U=\alpha F_{C}{ }^{\beta} N^{\gamma}=735.5 F_{C}^{1.095} N^{0.405} .
$$

\section{Theory of Two-Layer Predictive Control}

In modern process industries, the MPC controller is part of a multilevel hierarchy of optimization and control functions. Typically it is three-layer structure; that is, an RTO block is at the top layer, a MPC block is at the middle, and a PID block is at the bottom [19]. Therefore, under this multilevel hierarchy control system structure, the primary task of the MPC is to dynamic track the computational target calculated by the RTO. RTO layer should be optimized for the whole device.

Reference [20] proposed the framework of two-layer predictive control shown in Figure 2. SSO is added between RTO and MPC. Left branch, the SSO layer is used for recalculating the results of RTO layer, make the output steady-state target be located in the steady state gain matrix column space, so as to meet the compatibility and consistency 
conditions of steady state solution. Right branch, the role of SSO is to conduct local optimization to further improve the MPC steady-state performance, which can effectively resolve the nonparty system setpoints in the given problem.

Mathematical description of the two-layer predictive control include establishing steady-state mathematical model, steady-state target calculation, and a dynamic controller design [21].

3.1. Establish Steady-State Mathematical Model. Assume an MIMO plant with $m$ control input and $p$ controlled output and the coefficients of the corresponding step response model between control input $u_{j}$ and output $y_{i}$ are given; the model vector is

$$
a_{i j}(t)=\left[a_{i j}(1), \ldots, a_{i j}(N)\right]^{T},
$$

where $i=1, \ldots, p ; j=1, \ldots, m . N$ in (11) denotes modeling horizon of step response model. Thus, a multistep predictive model can be obtained:

$$
y(k+1)=y(k)+A_{1} \Delta u(k)
$$

where

$$
\begin{gathered}
y(k+1)=\left[\begin{array}{c}
y_{1}(k+1) \\
\vdots \\
y_{p}(k+1)
\end{array}\right] ; \quad y(k)=\left[\begin{array}{c}
y_{1}(k) \\
\vdots \\
y_{p}(k)
\end{array}\right] ; \\
\Delta u(k)=\left[\begin{array}{c}
\Delta u_{1}(k) \\
\vdots \\
\Delta u_{m}(k)
\end{array}\right] ; \quad A_{1}=\left[\begin{array}{ccc}
a_{11}(1) & \cdots & a_{1 m}(1) \\
\vdots & \vdots & \vdots \\
a_{p 1}(1) & \cdots & a_{p m}(1)
\end{array}\right] .
\end{gathered}
$$

Under the control increment $\Delta u(k), \ldots, \Delta u(k+M-1)$ action, the output predictive value of the system is

$$
\begin{gathered}
y(k+1)=y(k)+A_{1} \Delta u(k), \\
y(k+2)=y(k)+A_{2} \Delta u(k)+A_{1} \Delta u(k+1), \\
\vdots \\
y(k+N)=y(k)+A_{N} \Delta u(k)+\cdots \\
+A_{N-M+1} \Delta u(k+M-1),
\end{gathered}
$$

abbreviated as

$$
\partial y(k)=A \Delta u_{M}(k)
$$

where

$$
\partial y(k)=\left[\begin{array}{c}
y(k+1)-y(k) \\
\vdots \\
y(k+N)-y(k)
\end{array}\right],
$$

$$
\begin{gathered}
\Delta u_{M}(k)=\left[\begin{array}{c}
\Delta u(k) \\
\vdots \\
\Delta u(k+M-1)
\end{array}\right], \\
A=\left[\begin{array}{ccc}
A_{1} & 0 \\
\vdots & \ddots & \\
A_{M} & \cdots & A_{1} \\
\vdots & & \vdots \\
A_{N} & \cdots & A_{N-M+1}
\end{array}\right] .
\end{gathered}
$$

The system can be written at the steady-state time

$$
\Delta y(\infty)=A_{N} \Delta u(\infty),
$$

where $\Delta y(\infty)=\left[\Delta y_{1}(\infty), \Delta y_{2}(\infty), \ldots, \Delta y_{p}(\infty)\right]^{T}, \Delta u(\infty)=$ $\left[\Delta u_{1}(\infty), \Delta u_{2}(\infty), \ldots, \Delta u_{m}(\infty)\right]^{T}$ are the steady-state output increment and input increment, respectively, and $A_{N}$ is the steady-state step response coefficients matrix

$$
A_{N}=\left[\begin{array}{ccc}
a_{11}(N) & \cdots & a_{1 m}(N) \\
\vdots & \vdots & \vdots \\
a_{p 1}(N) & \cdots & a_{p m}(N)
\end{array}\right] .
$$

To meet the requirements of steady-state target calculation, model (17) can also be written as

$$
\Delta y_{\infty}(k)=A_{N} \Delta u_{\infty}(k)
$$

\subsection{Steady-State Target Calculation}

3.2.1. Basic Problem Description. Steady-state target calculation is to maximize economic benefits for the purpose of self-optimization under MPC existing configuration mode according to the process conditions. According to the production process characteristics and objectives, the basic problem of steady-state target calculation is the optimization process, which controlled input as cost variables, controlled output as steady-state variables. A common description of the objective function is as follows [21]:

$$
\min _{\Delta u_{\infty}(k), \Delta y_{\infty}(k)} J=\alpha^{T} \Delta u_{\infty}(k)+\beta^{T} \Delta y_{\infty}(k) .
$$

Since $\Delta u_{\infty}$ and $\Delta y_{\infty}$ are linearly related, the input output variation of objective function can be unified to control the input change. The formula (20) can be unified as

$$
\min _{\Delta u_{\infty}(k)} J=c^{T} \Delta u_{\infty}(k),
$$

where $c^{T}=\left[c_{1}, \ldots, c_{m}\right]$ is the cost coefficient vector, constructed by the normalized benefit, or cost of each input variable. $\Delta u_{\infty}(k)=\left[\Delta u_{\infty}^{1}, \ldots, \Delta u_{\infty}^{m}\right]^{T}$ is the steady-state change value of every input at time $k$.

Given the steady-state constraints of input and output variables, global-optimization problem of steady-state target 
calculation can be described as the following linear program (LP) problem:

$$
\begin{array}{cl}
\min _{\Delta u_{\infty}(k)} & J=c^{T} \Delta u_{\infty}(k) \\
\text { s.t. } & \Delta y_{\infty}(k)=G_{u} \Delta u_{\infty}(k)+G_{f} \Delta f_{\infty}(k)+e, \\
& u_{\min } \leq u_{\infty}(k)+\Delta u_{\infty}(k) \leq u_{\max }, \\
& y_{\min } \leq y_{\infty}(k)+\Delta y_{\infty}(k) \leq y_{\max },
\end{array}
$$

where $G_{u}, G_{f}$ are the steady-state gain matrices of control input and disturbance variables; and $e$ is the model bias. $u_{\min }, u_{\max }$ are low limit and upper limit of steady-state input variables $y_{\min }, y_{\max }$ are low limit and upper limit of steady state output variables.

The global-optimization problem of steady-state target calculation can be described as the following quadratic program $(\mathrm{QP})$ problem:

$$
\begin{array}{cl}
\min _{\Delta u_{\infty}(k)} & J=c^{T}\left(\Delta u_{\infty}(k)-\text { Maxprofit }\right)^{2} \\
\text { s.t. } & \Delta y_{\infty}(k)=G_{u} \Delta u_{\infty}(k)+G_{f} \Delta f_{\infty}(k)+e, \\
& u_{\min } \leq u_{\infty}(k)+\Delta u_{\infty}(k) \leq u_{\max }, \\
& y_{\min } \leq y_{\infty}(k)+\Delta y_{\infty}(k) \leq y_{\max },
\end{array}
$$

where Maxprofit is the potential maximum economic profit.

3.2.2. Feasibility Judgment and Soft Constraint Adjustment. Mathematically, optimization feasibility is the existence problem of the optimal solution. Feasibility of steady-state target calculation means that optimal steady state of input-output should meet their operating constraints; if feasible solution does not exist, the optimization calculation has no solution. The solving process is as follows: first, judge the existence of space domain formed by the constraints and if there is in it for optimization, if does not exist, then through the soft constraints adjustment to obtain the feasible space domain, and then to solve.

Soft constraints adjustment is an effective way to solve infeasible optimization [22, 23]. By relaxing the output constraints within the hard constraints, increasing the optimization problem feasible region that feasible solution to be optimized. Hard constraints refer to unalterable constraints limited by the actual industrial process.

Engineering standards of the priority strategy of soft constraints adjustment are the following: give priority to meet the highly important operating constraints, and allow less important operating constraints to be violated appropriately under the premise of satisfying the engineering constraints.

Considering the following constraints (24), constituted by steady-state model input constraints and output constraints containing slack variables, the priority rank is " $N$ ", where

$$
\begin{gathered}
\Delta y_{\infty}(k)=G_{u} \Delta u_{\infty}(k)+G_{f} \Delta f_{\infty}(k)+e, \\
u_{L L} \leq u_{\infty}(k)+\Delta u_{\infty}(k) \leq u_{H L}, \\
y_{L L}^{j}-\varepsilon_{2}^{j} \leq y_{\infty}(k)+\Delta y_{\infty}(k) \leq y_{H L}^{j}+\varepsilon_{1}^{j}, \\
\varepsilon_{1}^{j} \geq 0, \quad \varepsilon_{2}^{j} \geq 0, \\
\varepsilon_{1}^{j} \leq y_{H H L}-y_{H L}, \\
\varepsilon_{2}^{j} \leq y_{L L}-y_{L L L}, \\
j=1, \ldots, N .
\end{gathered}
$$

The algorithm steps of feasibility judgment and soft constraint adjustment based on the priority strategy are as follows.

Step 1. Initialization: according to the characteristics of the output variables and process conditions, set the upper and lower output constraints priority ranks, the same priority rank setting adjustments according to actual situation constraint weights.

Step 2. According to the priority ranks, judge the feasibility and adjust the soft constraints in accordance with the ranks from large to small. Under a larger priority rank if cannot find a feasible solution, the constraints of the rank will be relaxed to hard constraints, and then consider less priority rank constraints, until we find a feasible solution.

Step 3. Then the steady-state target calculation entered the stage of economy optimization or target tracking.

For Step 2, constraints of the highest priority rank $N$ are adjusted first by solving the following optimization problem:

$$
\begin{array}{ll}
\min _{\varepsilon^{N}} & J=\left(W^{N}\right)^{T} \varepsilon^{N}, \quad\left(W^{N}\right)^{T}=\left[W_{1}^{N}, \ldots, W_{2 \times n_{N}}^{N}\right] \\
\text { s.t. } & \Theta^{N} Z^{N}=b^{N}, \\
& \Omega^{N} Z^{N} \leq \Psi^{N},
\end{array}
$$

where

$$
\begin{gathered}
Z^{N}=\left[X_{1}^{T}, X_{2}^{T},\left(X_{3}^{1}\right)^{T}, \ldots,\left(X_{3}^{N}\right)^{T},\left(X_{4}^{1}\right)^{T}, \ldots,\right. \\
\left.\left(X_{4}^{N}\right)^{T},\left(\varepsilon_{1}^{N}\right)^{T},\left(\varepsilon_{2}^{N}\right)^{T},\left(\varepsilon_{1}^{N}\right)^{T},\left(\varepsilon_{2}^{N}\right)^{T}\right]^{T}, \\
\Omega^{N}=\operatorname{block}-\operatorname{diag}\left(-I_{m},-I_{m},-I_{n_{1}}, \ldots,-I_{n_{N}},\right. \\
\left.-I_{n_{1}}, \ldots,-I_{n_{N}},-I_{n_{N}},-I_{n_{N}}, I_{n_{N}}, I_{n_{N}}\right),
\end{gathered}
$$




$$
\begin{aligned}
& \Psi^{N}=\left[\left(0_{m \times 1}\right)^{T},\left(0_{m \times 1}\right)^{T},\left(0_{n_{1} \times 1}\right)^{T}, \ldots,\left(0_{n_{N} \times 1}\right)^{T}\right. \\
& \left(0_{n_{1} \times 1}\right)^{T}, \ldots,\left(0_{n_{N} \times 1}\right)^{T},\left(0_{n_{N} \times 1}\right)^{T},\left(0_{n_{N} \times 1}\right)^{T}, \\
& \left.\left(y_{H H L}^{N}-y_{H L}^{N}\right)^{T},\left(y_{L L}^{N}-y_{L L L}^{N}\right)^{T}\right]^{T} \text {, } \\
& b^{N}=\left[\begin{array}{c}
u_{H L}-u_{L L} \\
G_{u}^{1} u_{\infty}(k)-G_{u}^{1} u_{L L}(k)+y_{H L}^{1}-y_{\infty}^{1}(k)-G_{f}^{1} \Delta f_{\infty}(k)-e^{1} \\
\vdots \\
G_{u}^{N} u_{\infty}(k)-G_{u}^{N} u_{L L}(k)+y_{H L}^{N}-y_{\infty}^{N}(k)-G_{f}^{N} \Delta f_{\infty}(k)-e^{N} \\
G_{u}^{1} u_{H L}-G_{u}^{1} U_{\infty}(k)+Y_{\infty}^{1}(k)+G_{f}^{1} \Delta f_{\infty}(k)-y_{L L}^{1}+e^{1} \\
\vdots \\
G_{u}^{N} u_{H L}-G_{u}^{N} U_{\infty}(k)+y_{\infty}^{N}(k)+G_{f}^{N} \Delta f_{\infty}(k)-y_{L L}^{N}+e^{N} \\
0 \\
0
\end{array}\right], \\
& \Theta^{N}=\left[\begin{array}{cccccccccccc}
I_{m} & I_{m} & 0 & \cdots & 0 & 0 & \cdots & 0 & 0 & 0 & 0 & 0 \\
G_{u}^{1} & 0 & 0 & \cdots & 0 & I_{n_{1}} & 0 & 0 & 0 & 0 & 0 & 0 \\
\vdots & \vdots & 0 & \cdots & 0 & 0 & \ddots & 0 & 0 & 0 & 0 & 0 \\
G_{u}^{N} & 0 & 0 & \cdots & 0 & 0 & 0 & I_{n_{N}} & -I_{n_{N}} & 0 & 0 & 0 \\
0 & G_{u}^{1} & I_{n_{1}} & 0 & 0 & 0 & \cdots & 0 & 0 & 0 & 0 & 0 \\
\vdots & \vdots & 0 & \ddots & 0 & 0 & \cdots & 0 & 0 & 0 & 0 & 0 \\
0 & G_{u}^{N} & 0 & 0 & I_{n_{N}} & 0 & \cdots & 0 & 0 & -I_{n_{N}} & 0 & 0 \\
0 & 0 & 0 & \cdots & 0 & 0 & \cdots & 0 & I_{n_{N}} & 0 & -I_{n_{N}} & 0 \\
0 & 0 & 0 & \cdots & 0 & 0 & \cdots & 0 & 0 & I_{n_{N}} & 0 & -I_{n_{N}}
\end{array}\right] .
\end{aligned}
$$

Solving (25) may appear in three different cases, respectively: if (25) is feasible, and the optimum solution is $\varepsilon^{N}=0$, subject to $J=0$, that is, no need for soft constraints adjustment, directly solve the original problem (22); if (25) is feasible, but $\varepsilon^{N} \neq 0$, just need to relax constraints of priority ranks $N$, and further optimization solution; if (25) is infeasible, not get a feasible solution to soft constraints adjustment of the priority $\operatorname{rank} N$, relaxing the constraints of the priority $\operatorname{rank} N$ to hard constraints; that is,

$$
\begin{gathered}
\varepsilon_{1}^{N}=y_{H H L}^{N}-y_{H L}^{N}, \\
\varepsilon_{2}^{N}=y_{L L}^{N}-y_{L L L}^{N} .
\end{gathered}
$$

Go to the procedure of judging rank $N-1$ constraints

$$
\begin{array}{ll}
\min _{\varepsilon^{N-1}} & J=\left(W^{N-1}\right)^{T} \varepsilon^{N-1},\left(W^{N-1}\right)^{T}=\left[W_{1}^{N-1}, \ldots, W_{2 \times n_{N-1}}^{N-1}\right] \\
\text { s.t. } & \Theta^{N-1} Z^{N-1}=b^{N-1}, \\
& \Omega^{N-1} Z^{N-1} \leq \Psi^{N-1} .
\end{array}
$$

For (28), the matrix form is the same with priority rank $N$, only in the corresponding position of $\varepsilon^{N-1}$ to replace $\varepsilon^{N}, b^{N-1}$ matrix is adjusted

$$
b^{N-1}=\left[\begin{array}{c}
u_{H L}-u_{L L} \\
G_{u}^{1} u_{\infty}(k)-G_{u}^{1} u_{L L}(k)+y_{H L}^{1}-y_{\infty}^{1}(k)-G_{f}^{1} \Delta f_{\infty}(k)-e^{1} \\
\vdots \\
G_{u}^{N} u_{\infty}(k)-G_{u}^{N} u_{L L}(k)+y_{H L}^{N}-y_{\infty}^{N}(k)-G_{f}^{N} \Delta f_{\infty}(k)-e^{N}+\left(y_{H H L}^{N}-y_{H L}^{N}\right)^{T} \\
G_{u}^{1} u_{H L}-G_{u}^{1} u_{\infty}(k)+y_{\infty}^{1}(k)+G_{f}^{1} \Delta f_{\infty}(k)-y_{L L}^{1}+e^{1} \\
\vdots \\
G_{u}^{N} u_{H L}-G_{u}^{N} u_{\infty}(k)+y_{\infty}^{N}(k)+G_{f}^{N} \Delta f_{\infty}(k)-y_{L L}^{N}+e^{N}+\left(y_{L L}^{N}-y_{L L L}^{N}\right)^{T} \\
0 \\
0
\end{array}\right] .
$$


$N-1$ rank and $N$ rank are the same for the soft constraints adjustment processing, until the end of constraint adjustment of the priority rank 1 . If all ranks of constraints are relaxed to the hard constrain and a feasible solution still can't be found, then the original problem of soft constraints adjustment is infeasible and needs to be redesigned.

3.3. Dynamic Controller Design. In engineering applications, dynamic matrix control (DMC) algorithm is one of the most widely used MPC algorithms based on the step response model of the plant. This paper adopts DMC and steady-state target calculation integration strategy.

The difference is that the general DMC algorithms have no requirements on the steady-state position of the control input, and they only require the controlled output as close as possible to arrive at its set point. However, the integration strategy DMC requires both input and output variables to approach their steady-state targets $\left(\mathbf{u}_{s}, \mathbf{y}_{s}\right)$ as far as possible. The algorithm has three basic characteristics: predictive model, receding horizon optimization, and feedback correction [24].

3.3.1. Predictive Model. Based on system process step response model, at the current time $k$, the future $P$-step prediction output can be written as follows:

$$
\tilde{\mathbf{y}}_{P M}(k)=\tilde{\mathbf{y}}_{P 0}(k)+\mathbf{A} \Delta \mathbf{u}_{M}(k),
$$

where $P$ denotes the prediction horizon, $M$ is the control horizon, $\mathbf{A}$ is the prediction matrix composed by the corresponding step response coefficients, $\widetilde{\mathbf{y}}_{P 0}$ is the initial output prediction value when control action starting from the present time does not change, $\Delta \mathbf{u}_{M}(k)$ is the prediction incremental in $M$ control horizon, and $\widetilde{\mathbf{y}}_{P M}(k)$ is the future $P$ step prediction output under $M$-step control action change. Among them

$$
\begin{array}{cc}
\tilde{\mathbf{y}}_{P M}(k)=\left[\begin{array}{c}
\tilde{y}_{1, P M}(k) \\
\vdots \\
\tilde{y}_{p, P M}(k)
\end{array}\right], & \tilde{\mathbf{y}}_{P 0}(k)=\left[\begin{array}{c}
\tilde{y}_{1, P 0}(k) \\
\vdots \\
\tilde{y}_{p, P 0}(k)
\end{array}\right], \\
\Delta \mathbf{u}_{M}(k)=\left[\begin{array}{c}
\Delta u_{1, M}(k) \\
\vdots \\
\Delta u_{m, M}(k)
\end{array}\right], & \mathbf{A}=\left[\begin{array}{ccc}
A_{11} & \cdots & A_{1 m} \\
\vdots & \ddots & \vdots \\
A_{p 1} & \cdots & A_{p m}
\end{array}\right] .
\end{array}
$$

3.3.2. Receding Horizon Optimization. In the receding horizon optimization process, control increment can be obtained in every execution cycle by minimizing the following performance index:

$$
\begin{aligned}
\min _{\Delta \mathbf{u}_{M}(k)} J(k)= & \left\|\mathbf{w}(k)-\widetilde{\mathbf{y}}_{P M}(k)\right\|_{\mathbf{Q}}^{2}+\|\boldsymbol{\varepsilon}(k)\|_{\mathbf{S}}^{2} \\
& +\left\|\mathbf{u}_{M}(k)-\mathbf{u}_{\infty}\right\|_{\mathbf{T}}^{2}+\left\|\Delta \mathbf{u}_{M}(k)\right\|_{\mathbf{R}}^{2} .
\end{aligned}
$$

Subject to the model

$$
\tilde{\mathbf{y}}_{P M}(k)=\widetilde{\mathbf{y}}_{P 0}(k)+\mathbf{A} \Delta \mathbf{u}_{M}(k) .
$$

Subject to bound constraints

$$
\mathbf{y}_{\min }-\boldsymbol{\varepsilon} \leq \widetilde{\mathbf{y}}_{P M}(k) \leq \mathbf{y}_{\max }+\boldsymbol{\varepsilon},
$$

$$
\begin{gathered}
\mathbf{u}_{\min } \leq \mathbf{u}_{M} \leq \mathbf{u}_{\max }, \\
\Delta \mathbf{u}_{\min } \leq \Delta \mathbf{u}_{M}(k) \leq \Delta \mathbf{u}_{\max },
\end{gathered}
$$

where $\boldsymbol{\varepsilon}$ denotes the slack variables, guaranteeing the feasibility of the DMC optimization, and $w(k)=\left[w_{1}(k), \ldots, w_{p}(k)\right]^{T}$ is the setpoint of controlled output obtained from upper SSO layer. $\mathbf{Q}, \mathbf{R}$ are the weight coefficient matrix

$$
\begin{gathered}
\mathbf{Q}=\text { block-diag }\left(Q_{1}, \ldots, Q_{p}\right), \\
\mathbf{Q}_{i}=\operatorname{diag}\left(q_{i}(1), \ldots, q_{i}(P)\right), \quad i=1, \ldots, p, \\
\mathbf{R}=\text { block-diag }\left(R_{1}, \ldots R_{m}\right), \\
\mathbf{R}_{j}=\operatorname{diag}\left(r_{i}(1), \ldots, r_{i}(M)\right), \quad j=1, \ldots, m .
\end{gathered}
$$

Through the necessary conditions of extreme value $\partial J / \partial \Delta u_{M}(k)=0$, the optimal increment of control input can be obtained:

$$
\Delta \mathbf{u}_{M}(k)=\left(\mathbf{A}^{T} \mathbf{Q A}+\mathbf{R}\right)^{-1} \mathbf{A}^{T} \mathbf{Q}\left[\mathbf{w}(k)-\widetilde{\mathbf{y}}_{P 0}(k)\right] .
$$

The instant increment can be calculated as follows:

$$
\Delta \mathbf{u}(k)=\mathbf{L D}\left[\mathbf{w}(k)-\widetilde{\mathbf{y}}_{P 0}(k)\right],
$$

where $\mathbf{D}=\left(\mathbf{A}^{T} \mathbf{Q A}+\mathbf{R}\right)^{-1} \mathbf{A}^{T} \mathbf{Q}$; remark the operation of only the first element with

$$
L=\left[\begin{array}{ccccccccc}
1 & 0 & \cdots & 0 & & & & 0 & \\
& & & & \ddots & & & & \\
& & 0 & & & 1 & 0 & \cdots & 0
\end{array}\right]
$$

3.3.3. Feedback Correction. The difference between the process sample values by the present moment $k$ and prediction values of (30) is

$$
e(k+1)=\left[\begin{array}{c}
e_{1}(k+1) \\
\vdots \\
e_{p}(k+1)
\end{array}\right]=\left[\begin{array}{c}
y_{1}(k+1)-\tilde{y}_{1,1}(k+1 \mid k) \\
\vdots \\
y_{p}(k+1)-\tilde{y}_{p, 1}(k+1 \mid k)
\end{array}\right],
$$

where $\tilde{y}_{i, 1}(k+1 \mid k)$ is the first element of $\tilde{y}_{i, P M}(k+1 \mid k)$, and the corrected output prediction value can be obtained using the error vector; that is,

$$
\widetilde{\mathbf{y}}_{\mathrm{cor}}(k+1)=\widetilde{\mathbf{y}}_{N 1}(k)+\mathbf{H} e(k+1),
$$

where $\tilde{\mathbf{y}}_{N 1}(k)=\widetilde{\mathbf{y}}_{N 0}(k)+\mathbf{A}_{N} \Delta \mathbf{u}, \tilde{\mathbf{y}}_{N 0}(k)$ is the future $N$ moment initial prediction value when all of the input remained unchanged at the time $k ; \widetilde{\mathbf{y}}_{N 1}(k)$ is the future $N$ moment output prediction value under one-step control input action; $H$ is the error correct matrix. Then using a shift matrix $S$, next time the initial prediction value can be obtained, which is

$$
\tilde{y}_{N 0}(k+1)=S \tilde{y}_{\text {cor }}(k+1) \text {, }
$$




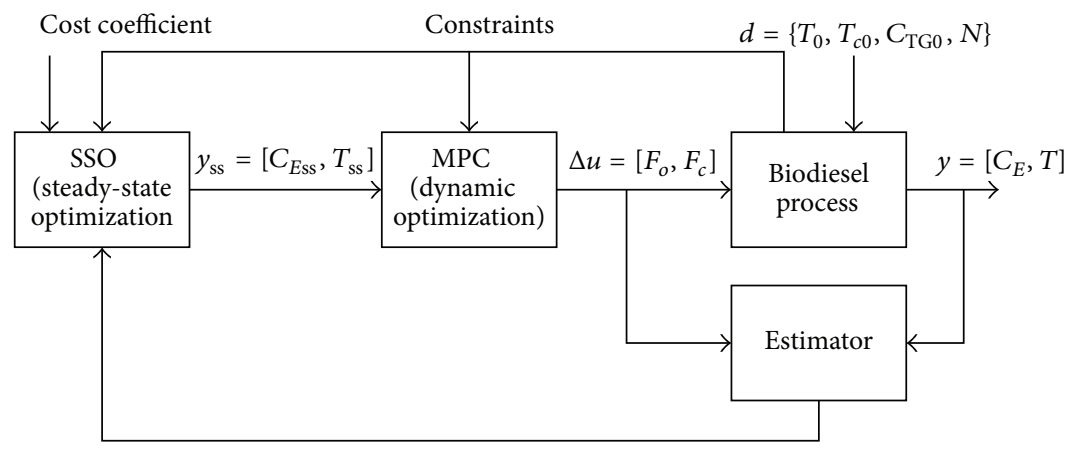

FIGURE 3: Two-layer predictive framework of biodiesel process.

where

$$
S=\left[\begin{array}{ccccc}
0 & 1 & & & 0 \\
& 0 & 1 & & \\
& & \ddots & \ddots & \\
& & & 0 & 1 \\
0 & & & & 1
\end{array}\right]_{N * N}
$$

\section{Control System Design}

In the biodiesel reactor control, multiloops are necessary to stabilize the plant. One loop is needed to maintain the set point of specifying the product purity, and another loop is needed to ensure an optimal yield of biodiesel and to minimize the generation of unwanted by-products even in the presence of disturbances.

To achieve these goals, the control loop configurations analysis is meaningful. Based on the analysis of Mjalli et al. [4], the favorable pairings are as follows: the biodiesel concentration $\left(C_{E}\right)$ is maintained by manipulating reactant flow rate $\left(F_{o}\right)$, the reactor temperature $(T)$ is maintained by manipulating coolant flow rate $\left(F_{c}\right)$, respectively, and the effect of stirred rotational speed on the reactor output is insignificant, and it would be regarded as one of disturbances to the control system. The relative gain array (RGA) shows that there are some interactions among the controlled and manipulated variables which make two-layer predictive controller better qualified.

Consequently, the two-layer predictive controller is designed to handle a $2 \times 2$ system of inputs and outputs. The controlled output variables include biodiesel concentration $\left(C_{E}\right)$ and reactor temperature $(T)$; the manipulated variables include reactant flow rate $\left(F_{o}\right)$ and coolant flow rate $\left(F_{c}\right)$. It is very important for a reactor to handle the disturbances in the feed concentration and initial temperatures, as these disturbances heavily change the system performance.

The design of the control loop based on the two-layer predictive control strategy for the biodiesel reactor is shown in Figure 3. The SSO layer searches the optimal output setpoints $C_{E s s}$ and $T_{s s}$ according to the economic optimization goal of the actual production process. The MPC layer selects the real-time control actions $\Delta u$ to complete the dynamic tracking control.

\section{Simulation Results and Analysis}

5.1. Model Identification. For the two-layer predictive control scheme to be successful, process modeling plays a key role in capturing the varying dynamics of the system. Section 4 shows that the biodiesel process is a two-input two-output multivariable process. The process nonlinear model was programmed and simulated in Matlab as a function. Simulation results show system is open stable process.

Firstly, generalized binary noise (GBN) signal is selected as the excitation signal. GBN signals switch between $a$ and $-a$ according to the following rules:

$$
\begin{gathered}
P[u(t)=-u(t-1)]=p_{s w}, \\
P[u(t)=u(t-1)]=1-p_{s w},
\end{gathered}
$$

where $p_{s w}$ is transition probability; $T_{\min }$ is defined as the sampling time of the signal held constant; $T_{s w}$ is time interval of twice conversion. The average conversion time and power spectrum are, respectively,

$$
\begin{gathered}
E T_{s w}=\frac{T_{\min }}{p_{s w}}, \\
\Phi_{u}(\omega)=\frac{\left(1-q^{2}\right) T_{\min }}{1-2 q \cos T_{\min } \omega+q^{2}}, \quad q=1-2 p_{s w} .
\end{gathered}
$$

Next, least squares (LS) identification method is used to estimate the process model parameters. Suppose an MIMO plant with $m$ input $p$ output, for the $i$ th output of the finite impulse response (FIR) model, is described as

$$
y_{i}(k)=\sum_{j=1}^{m} \sum_{l=1}^{N} h_{i j l} u_{j}(k-l) .
$$

Consider experimental tests of collecting input sequence

$$
\begin{array}{cccc}
u_{1}(1) & u_{1}(2) & \cdots & u_{1}(L) \\
\vdots & & & \vdots \\
u_{m}(1) & u_{m}(2) & \cdots & u_{m}(L)
\end{array}
$$

and output sequence

$$
\begin{array}{cccc}
y_{1}(1) & y_{1}(2) & \cdots & y_{1}(L) \\
\vdots & & & \vdots \\
y_{p}(1) & y_{p}(2) & \cdots & y_{p}(L) .
\end{array}
$$




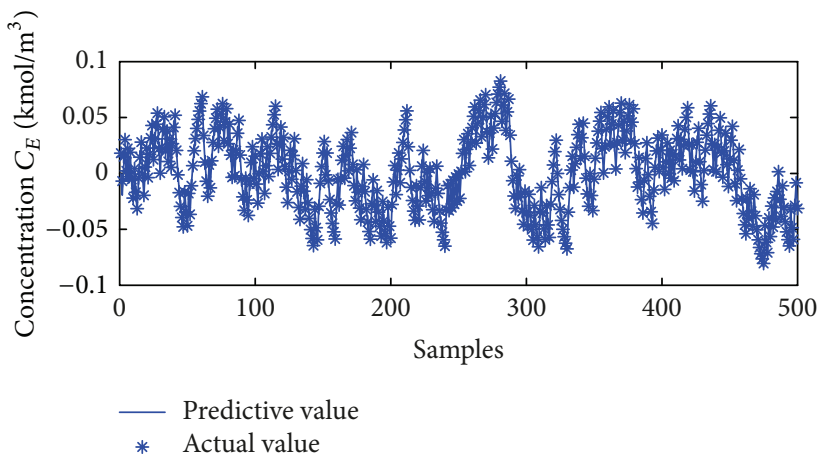

(a)

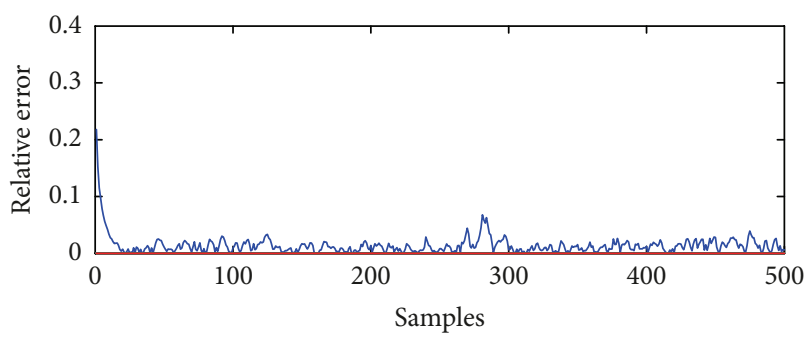

(b)

FIGURE 4: Biodiesel concentration prediction result and relative error under reactor flow rate $F_{o}$ action.

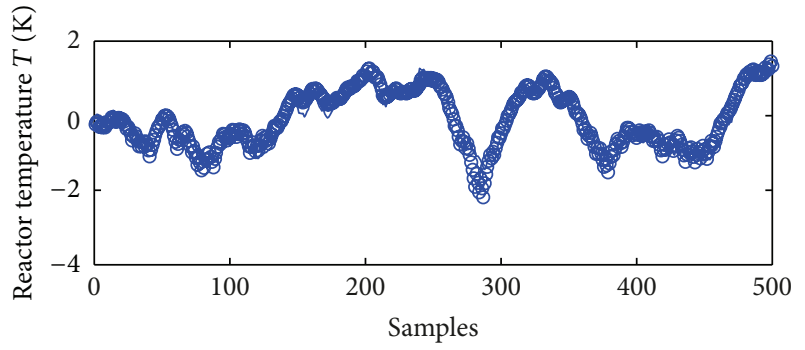

- Predictive value

- Actual value

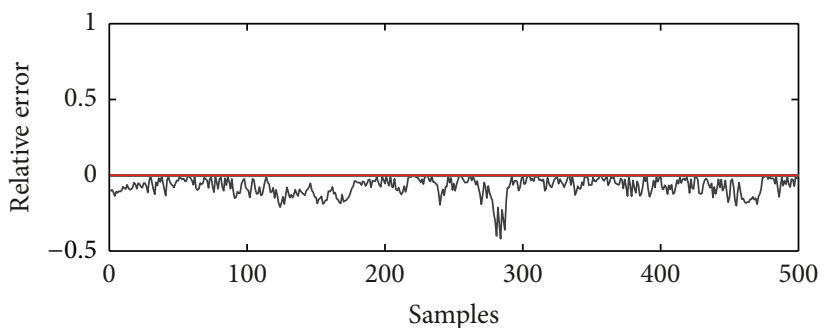

(b)

FIGURE 5: Reactor temperature prediction result and relative error under reactor flow rate $F_{o}$ action.

Consider matching between data and models; the introduction of residuals for each output can be independently expressed as follows:

$$
y_{i}(k)=\varphi(k) \theta_{i}+e(k) .
$$

$$
\begin{aligned}
& y_{i}=\left[\begin{array}{c}
y_{i}(N+1) \\
y_{i}(N+2) \\
\vdots \\
y_{i}(L)
\end{array}\right], \quad e=\left[\begin{array}{c}
e(N+1) \\
e(N+2) \\
\vdots \\
e(L)
\end{array}\right]
\end{aligned}
$$

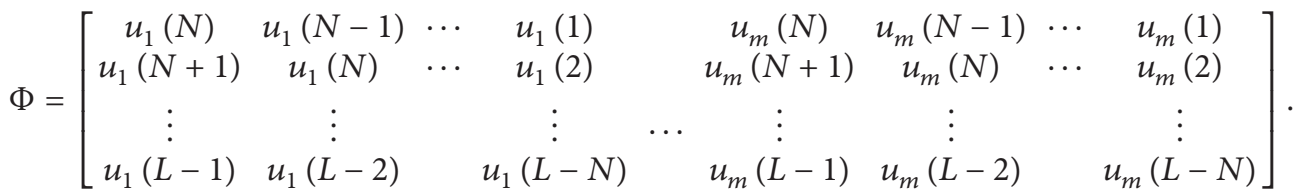

Minimize the squared residuals

$$
\min J=e^{T} e=[y-\Phi \theta]^{T}[y-\Phi \theta] .
$$

Obtain the optimal estimate

$$
\widehat{\theta}=\left[\Phi^{T} \Phi\right]^{-1} \Phi^{T} y
$$

Matrix form is written as

$$
y_{i}=\Phi \theta_{i}+e,
$$

where

For the model predictive controller design, the FIR model of system identification needs to be further converted into finite step response (FSR) model. The relationship between FSR coefficients and FIR coefficients is as follows:

$$
g_{j}=\sum_{i=1}^{j} h_{j} .
$$




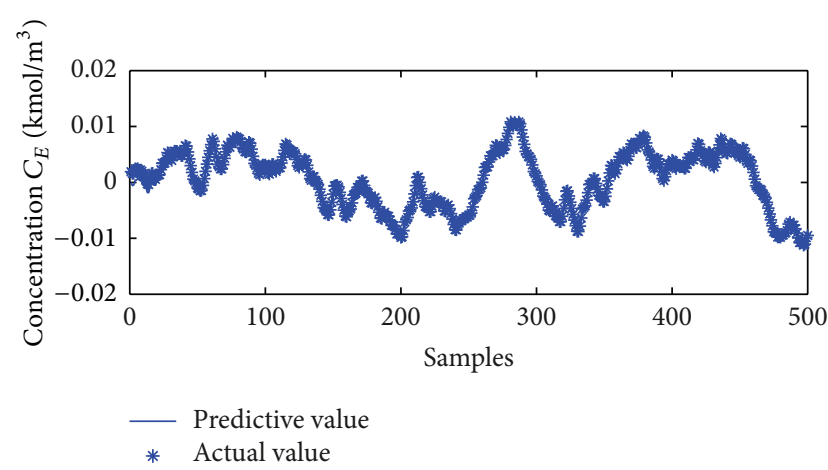

(a)

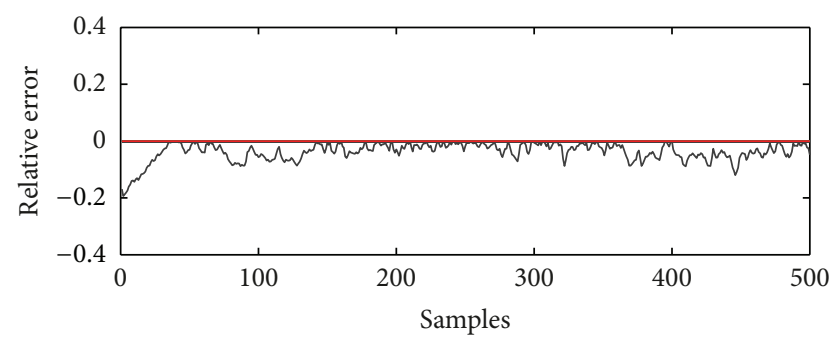

(b)

FIGURE 6: Biodiesel concentration prediction result and relative error under reactor flow rate $F_{c}$ action.

Coefficients matrix of FSR is

$$
G_{l}^{u}=\left[\begin{array}{cccc}
s_{11 l} & s_{12 l} & \cdots & s_{1 m l} \\
s_{21 l} & s_{22 l} & \cdots & s_{2 m l} \\
\vdots & \vdots & \ddots & \vdots \\
s_{p 1 l} & s_{p 2 l} & \cdots & s_{p m l}
\end{array}\right] .
$$

Finally, (11)-(19) are used to create a steady-state mathematical model of two-layer prediction control. The concrete simulation process is as follows.

In the work, GBN as the excitation signal was added to the model input to produce output data. The parameters of GBN signal applied to the first input are $T_{s w}=65, a m p=0.1$ the parameters of GBN applied to the second input are $T_{s w}=$ $65, a m p=0.005$, both the conversion probabilities are taken to be $P_{s w}=1 / T_{s w}$. Simulation time $t=2000 \mathrm{~s}$, and sample time equals $2 \mathrm{~s}$, under each input excitation, corresponding to two sets of output data each set of data capacity is 1000 . Among them, the former 500 data as model identification, the remaining data are used as model validations, and FSR model length value is taken as 200 .

Under the action of two inputs, reactant flow rate $F_{o}$ and coolant flow rate $F_{c}$, respectively, predicted value, actual value, and the relative error of two outputs biodiesel concentration $C_{E}$ and reactor temperature $T$ were shown in Figures $4,5,6$, and 7 . Figures $4-7$ show that relative error is small enough, and the model can describe $C_{E}$ and $T$ change trends under $F_{o}$ and $F_{c}$.

Figures 8 and 9 give the two output step response curves under two input $F_{o}, F_{c}$ action, respectively, further shows the multiple-input multiple-output system is open-loop stable

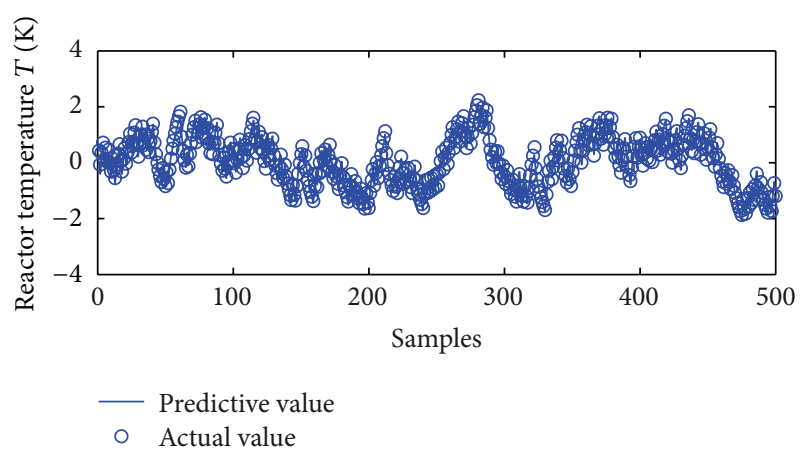

(a)

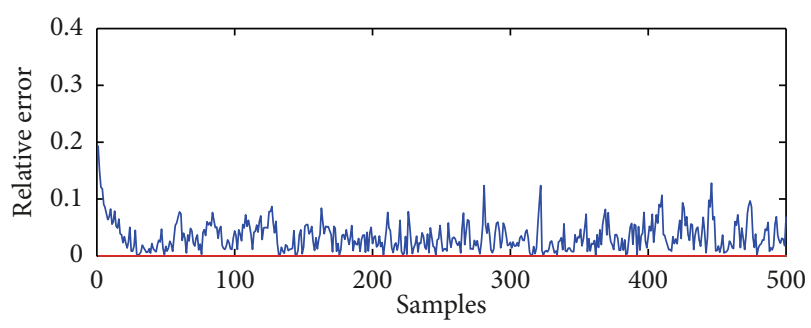

(b)

FIGURE 7: Reactor temperature prediction result and relative error under reactor flow rate $F_{c}$ action.

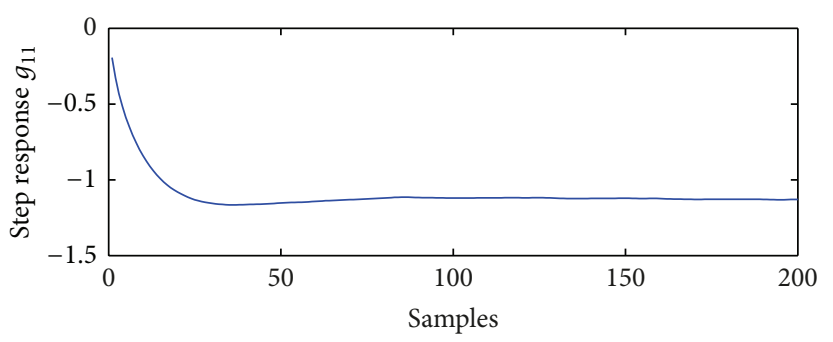

(a)

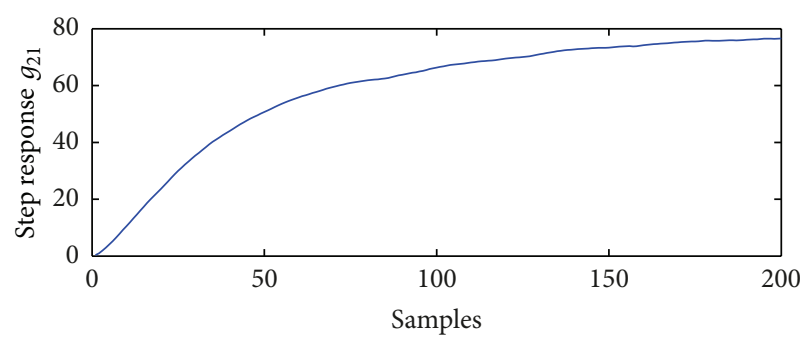

(b)

FIGURE 8: Step response curve of biodiesel concentration and reactor temperature, respectively, under $F_{o}$ action.

and the step response model has been identified successfully. The FSR model will be utilized to represent the actual process in latter optimization and controller design.

5.2. Dynamic Simulation. To validate the effectiveness and immunity in two-layer predictive control, the models obtained in Section 5.1 are used in the simulations. 


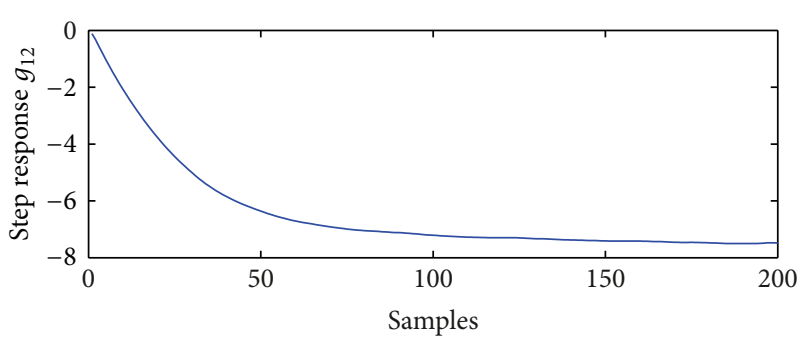

(a)

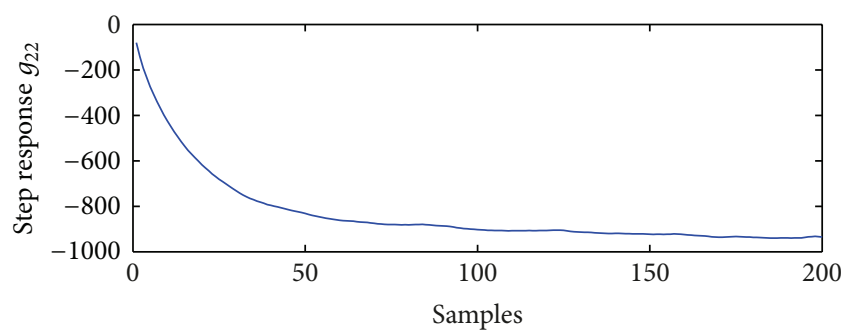

(b)

FIGURE 9: Step response curve of biodiesel concentration and reactor temperature, respectively, under $F_{c}$ action.

The reaction rate constants come from [18] under the common industrial conditions of $6: 1 \mathrm{methanol/oil} \mathrm{mole}$ ratio, $1.0 \mathrm{wt} \%$ catalyst $\mathrm{KOH}$, and $600 \mathrm{rpm}$ stirrer rotational speed. These kinetics parameters can be considered as constants. The initial operating conditions refer to the literature [4] the validated data. According to these parameters and reaction conditions, the simulation of biodiesel transesterification reactor can be carried out.

The economic optimization method described in (22) is adopted as SSO whose main parameters are selected as follows: the cost coefficients of control input in steady-state optimization are set to $[1 ;-1]$, the input $F_{o}$ is constrained between 0 and $0.2 \mathrm{~m}^{3} / \mathrm{s}$, the input $F_{c}$ is constrained between 0 and $0.1 \mathrm{~m}^{3} / \mathrm{s}$, and the output $C_{E}$ is constrained between $3.0536 \mathrm{kmol} / \mathrm{m}^{3}$ and $3.196 \mathrm{kmol} / \mathrm{m}^{3}$, the output $T$ is constrained between $337.77 \mathrm{~K}$ and $338.25 \mathrm{~K}$.

The parameters of the dynamic control layer adopted the unconstrained DMC algorithm: the modeling time domain $N=200$, prediction horizon $P=200$, control horizon $M=$ 20. The weight coefficient values of weight matrix $Q$ and $R$ equal to 10 and 1000 , respectively.

Conventional PID controller has also been designed in this simulation for comparison of performance to two-layer predictive controller. The parameters of PID controller for $C_{E}$ with $F_{o}$ control loop are $k p=-6 e-5, k i=-0.05$, and $k d=0$; the parameters for $T$ with $F_{c}$ control loop are $k p=-0.02$, $k i=-0.001$, and $k d=0$. The simulations of general PID controller and two-layer predictive controller are compared to validate the performance of the latter algorithm, whose results are shown in Figures 10 and 11.

As Figures 10 and 11 show, the two-layer predictive controller starts running at the time $t=0$. The results of steady state optimization are

$$
y_{s s}=[3.196,337.77], \quad u_{s s}=[0.073,0.0062] \text {. }
$$

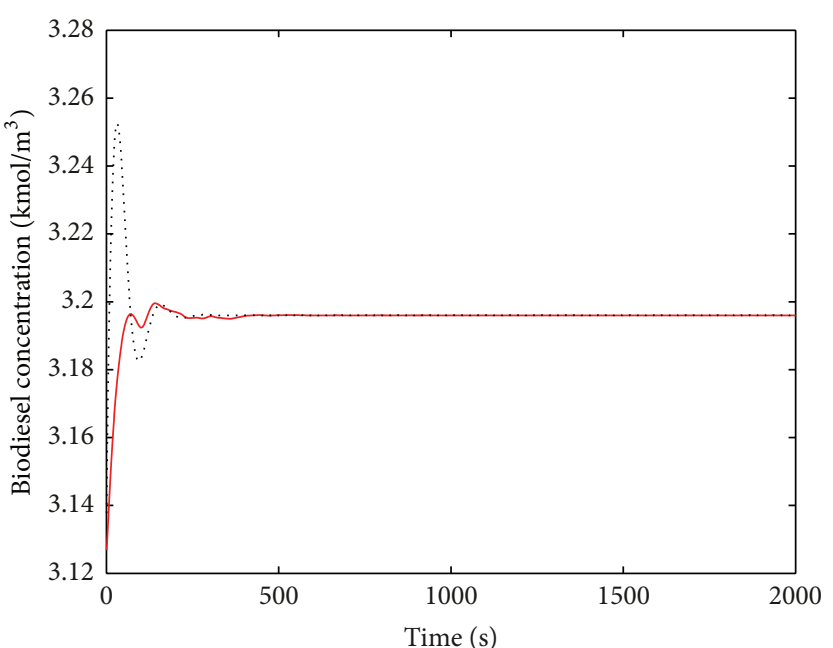

(a)

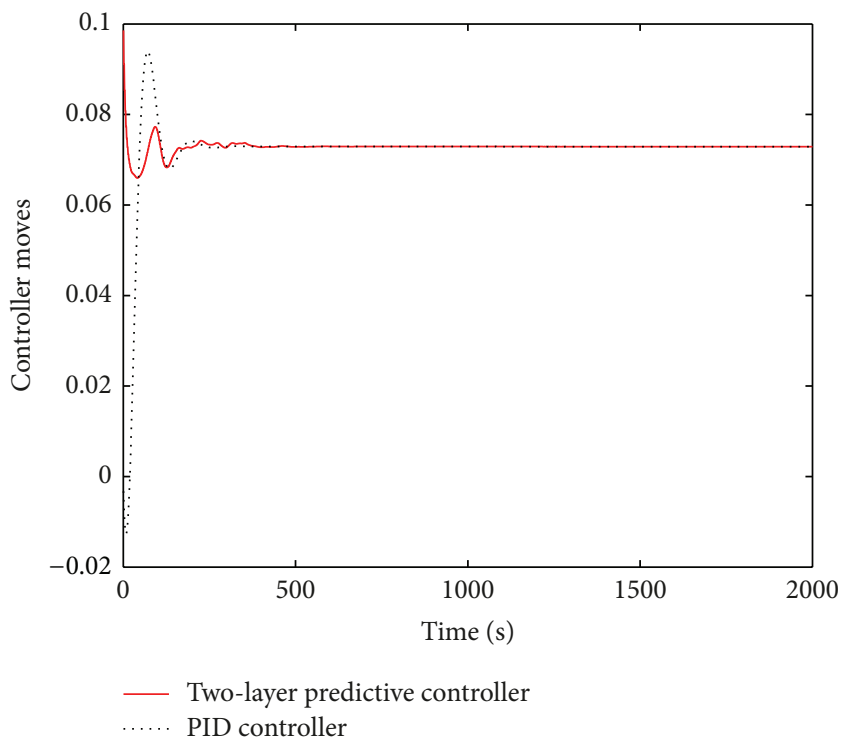

(b)

FIGURE 10: Biodiesel concentration and controller moves of twolayer predictive controller and PID controller.

The optimized values as the setpoints were send to the lower layer DMC. In the beginning, the closed loop response of the two-layer predictive controller was a little sluggish in bringing the biodiesel concentration back the optimum steady-state values, this is because that the algorithm enter the constraint adjustment stage based on the priority strategy which adjusting the upper limit and lower limit to be handled. About At the time $t=400$, the response gradually becomes stable. It can be seen that the two-layer predictive controller preceded the PID controller in terms of the ability to attain lower overshoot, smaller oscillation, and faster response time.

Considering the actual application, the control input is also an important indicator of good or bad controller. From Figures 10 and 11, the two-layer predictive controller has much more stable controller moves than does PID that meets the practical implementation constrains. 


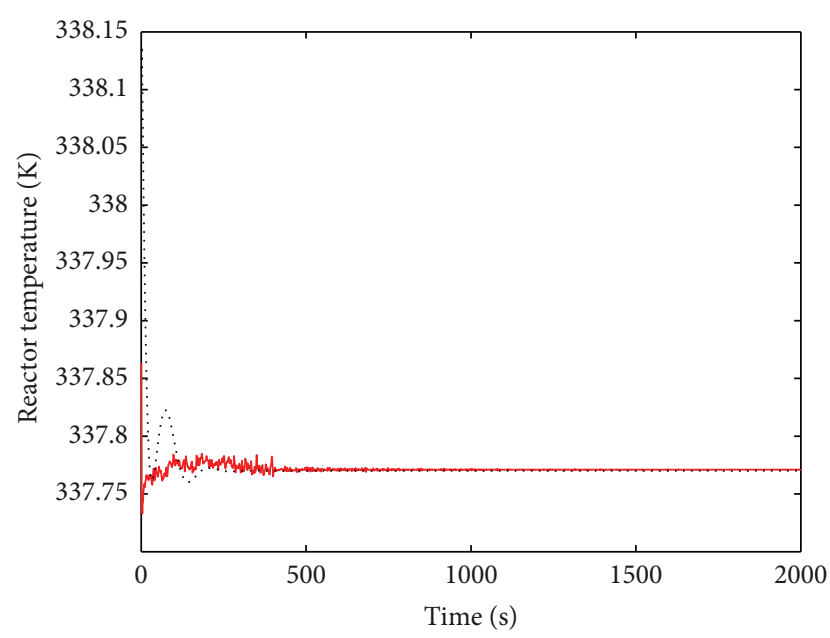

(a)

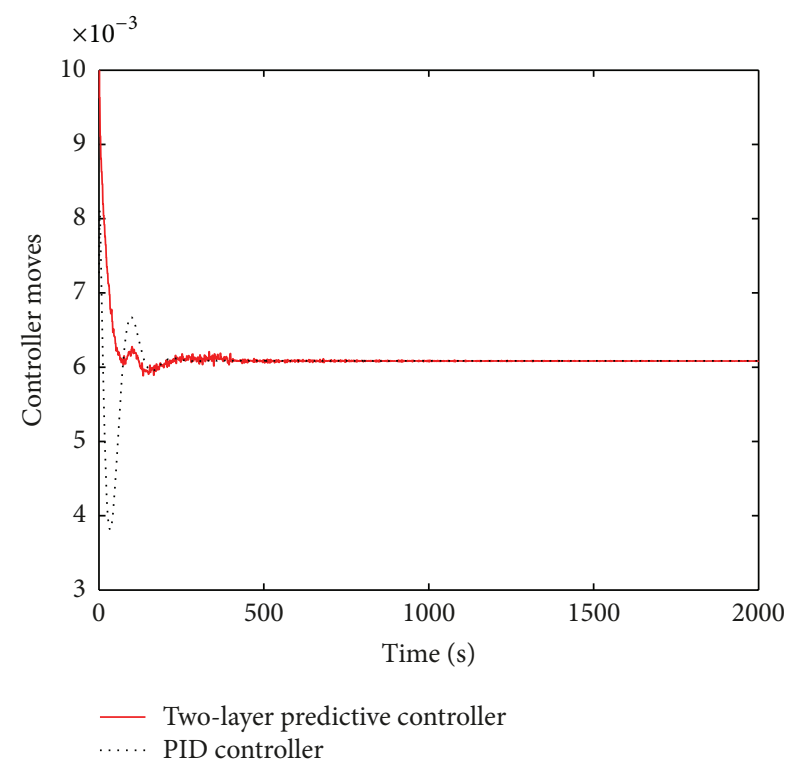

(b)

FIGURE 11: Reactor temperature and controller moves of two-layer predictive controller and PID controller.

To challenge the stability of two-layer predictive controller, some disturbances were exerted alone and at the same time. The chosen disturbance variables include coolant input temperature $\left(T_{c 0}\right)$, feed temperature $\left(T_{0}\right)$, triglyceride initial concentration $\left(C_{\mathrm{TG} 0}\right)$, and stirrer rotational speed $(N)$. After the system has attained the steady state, The nominal values of $T_{c 0}, T_{0}$ were increased $3 \mathrm{~K}$, respectively, and $C_{\mathrm{TG} 0}, N$ were increased 5\%, respectively, at the time $t=1000 \mathrm{~s}$. Figures 12 and 13 show the biodiesel concentration and reactor temperature profiles when these disturbance variables were introduced.

Figures 12 and 13 showed satisfactory rejection of all disturbances. Two-layer predictive controller was able to bring back the controlled variables to their setpoints in less than $1000 \mathrm{~s}$, and overshoot was within the acceptable range. For the biodiesel concentration loop, the initial concentration

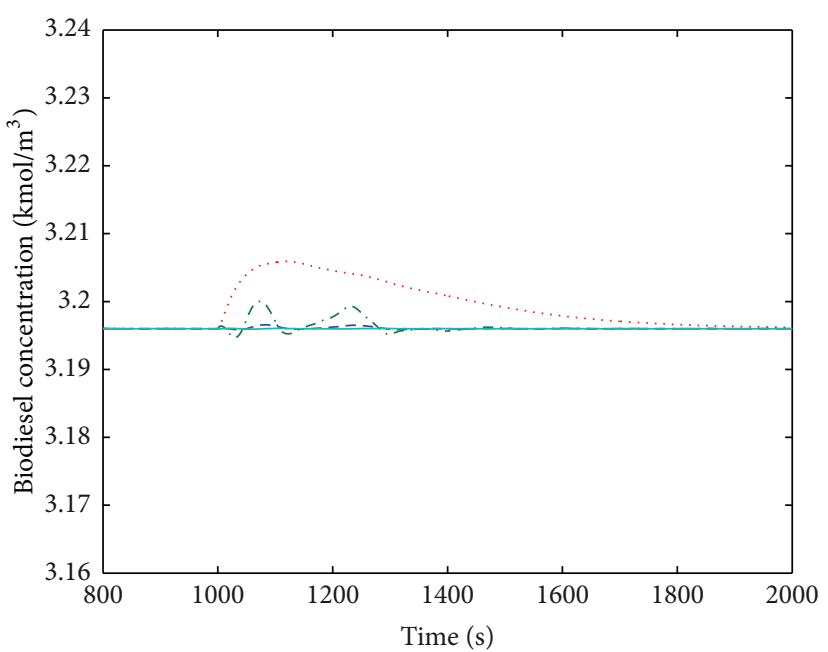

(a)

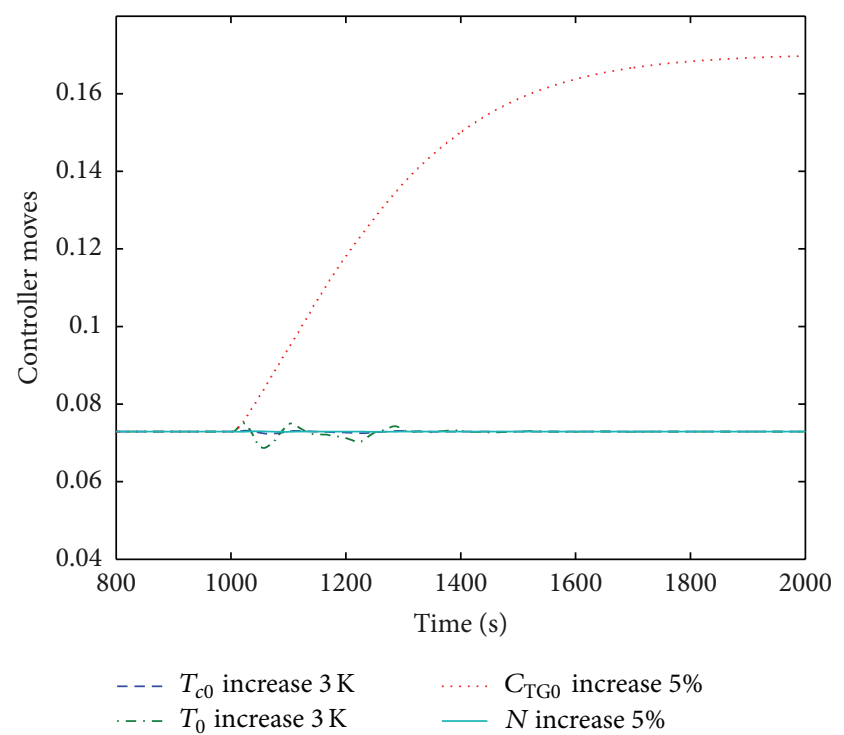

(b)

FIGURE 12: Biodiesel concentration and controller moves of four individual disturbance variables effects.

$C_{\mathrm{TG} 0}$ has the highest effect, with an overshoot of less than $0.01 \mathrm{kmol} / \mathrm{m}^{3}$. For the reactor temperature loop, the feed temperature $T_{0}$ has the largest effect, with an overshoot of less than $0.33 \mathrm{~K}$. For the two loops, the stirrer rotational speed almost has no effect on the controlled variables.

\section{Conclusions}

Biodiesel transesterification reactor control has become very important in recent years due to the difficulty in controlling the complex and highly nonlinear dynamic behavior. In this paper, a novel two-layer predictive control scheme for a continuous biodiesel transesterification reactor has been proposed. The SSO layer achieved optimal output setpoints according to the local economic optimization goal of the actual production process, and the MPC layer realized the 


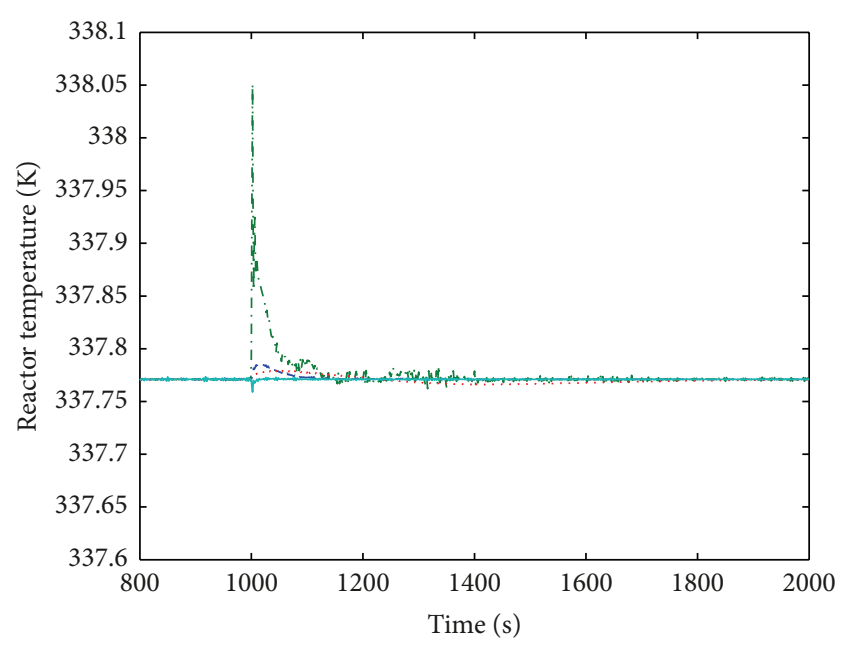

(a)

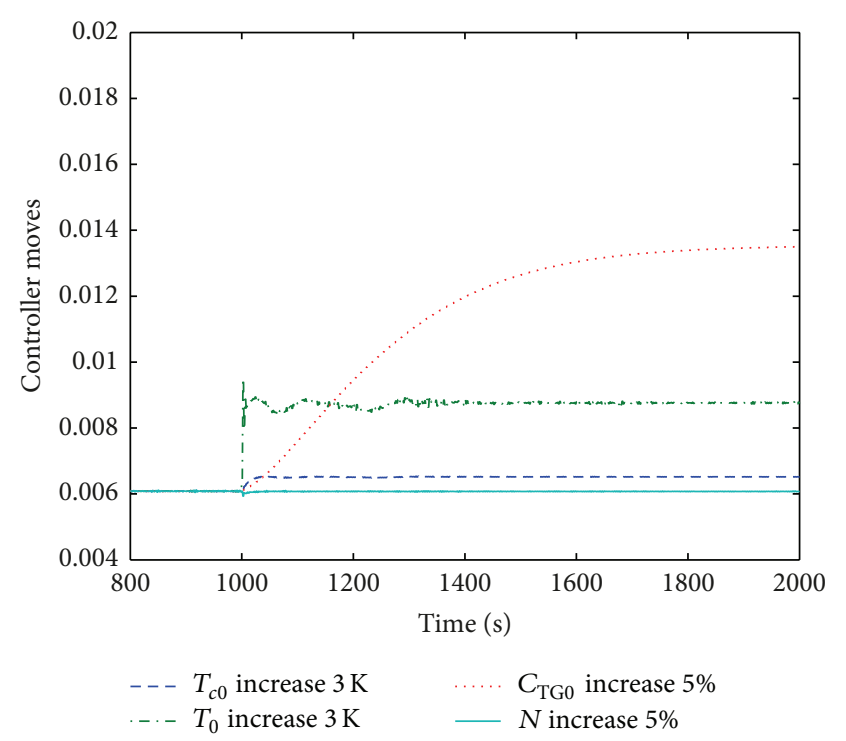

(b)

FIGURE 13: Reactor temperature and controller moves of four individual disturbance variables effects.

dynamic tracking control. The main aim was to optimize and control the biodiesel concentration and reactor temperature in order to obtain the product of the highest quality at the lower cost. With steady-state optimum target calculation and DMC algorithm implement, the performance of the two-layer predictive controller was superior to that of a conventional PID controller. The two-layer predictive control is not only stable but also tracks set points more efficiently with minimal overshoots and shorter settling times. Moreover, it exhibits good disturbance rejection characteristics.

\section{Acknowledgments}

This work is supported by the National Natural Science Foundation of China (61034008) and the Science Research
Foundation of Liaoning Provincial Department of Education (L2012145).

\section{References}

[1] D. Y. C. Leung, X. Wu, and M. K. H. Leung, "A review on biodiesel production using catalyzed transesterification," Applied Energy, vol. 87, no. 4, pp. 1083-1095, 2010.

[2] S. Shahla, N. G. Cheng, and R. Yusoff, "An overview on transesterification of natural oils and fats," Biotechnology and Bioprocess Engineering, vol. 15, no. 6, pp. 891-904, 2010.

[3] N. N. A. N. Yusuf, S. K. Kamarudin, and Z. Yaakub, "Overview on the current trends in biodiesel production," Energy Conversion and Management, vol. 52, no. 7, pp. 2741-2751, 2011.

[4] F. S. Mjalli, L. K. San, K. C. Yin, and M. A. Hussain, "Dynamics and control of a biodiesel transesterification reactor," Chemical Engineering and Technology, vol. 32, no. 1, pp. 13-26, 2009.

[5] T. Eevera, K. Rajendran, and S. Saradha, "Biodiesel production process optimization and characterization to assess the suitability of the product for varied environmental conditions," Renewable Energy, vol. 34, no. 3, pp. 762-765, 2009.

[6] C. S. Bildea and A. A. Kiss, "Dynamics and control of a biodiesel process by reactive absorption," Chemical Engineering Research and Design, vol. 89, no. 2, pp. 187-196, 2011.

[7] L. Zong, S. Ramanathan, and C.-C. Chen, "Fragment-based approach for estimating thermophysical properties of fats and vegetable oils for modeling biodiesel production processes," Industrial and Engineering Chemistry Research, vol. 49, no. 2, pp. 876-886, 2010.

[8] Y. K. Ho, F. S. Mjalli, and H. K. Yeoh, "Multivariable adaptive predictive model based control of a biodiesel transesterification reactor," Journal of Applied Sciences, vol. 10, no. 12, pp. 1019-1027, 2010.

[9] H. Y. Kuen, F. S. Mjalli, and Y. H. Koon, "Recursive least squaresbased adaptive control of a biodiesel transesterification reactor," Industrial and Engineering Chemistry Research, vol. 49, no. 22, pp. 11434-11442, 2010.

[10] W. A. Wali, A. I. Al-Shamma, K. H. Hassan, and J. D. Cullen, "Online genetic-ANFIS temperature control for advanced microwave biodiesel reactor," Journal of Process Control, vol. 22, pp. 1256-1272, 2012.

[11] P. T. Benavides and U. Diwekar, "Optimal control of biodiesel production in a batch reactor-part I: deterministic control," Fuel, vol. 94, pp. 211-217, 2012.

[12] S. J. Qin and T. A. Badgwell, "A survey of industrial model predictive control technology," Control Engineering Practice, vol. 11, no. 7, pp. 733-764, 2003.

[13] T. A. Johansen and A. Grancharova, "Approximate explicit constrained linear model predictive control via orthogonal search tree," IEEE Transactions on Automatic Control, vol. 48, no. 5, pp. 810-815, 2003.

[14] T. Zou, B. C. Ding, and D. Zhang, Model Predictive Control Engineering Applications Introduction, Chemical Industry Press, Beijing, China, 2010.

[15] A. Nikandrov and C. L. E. Swartz, "Sensitivity analysis of LPMPC cascade control systems," Journal of Process Control, vol. 19, no. 1, pp. 16-24, 2009.

[16] H. Noureddini and D. Zhu, "Kinetics of transesterification of soybean oil," Journal of the American Oil Chemists' Society, vol. 74, no. 11, pp. 1457-1463, 1997. 
[17] A.-F. Chang and Y. A. Liu, "Integrated process modeling and product design of biodiesel manufacturing," Industrial and Engineering Chemistry Research, vol. 49, no. 3, pp. 1197-1213, 2010.

[18] G. Vicente, M. Martínez, and J. Aracil, "Kinetics of Brassica carinata oil methanolysis," Energy and Fuels, vol. 20, no. 4, pp. 1722-1726, 2006.

[19] R. Scattolini, "Architectures for distributed and hierarchical Model Predictive Control-a review," Journal of Process Control, vol. 19, no. 5, pp. 723-731, 2009.

[20] T. Zou, H. Q. Li, B. C. Ding, and D. D. Wang, "Compatibility and uniqueness analyses of steady state solution for multi-variable predictive control systems," Acta Automatica Sinica, vol. 39, pp. 519-529, 2013.

[21] D. E. Kassmann, T. A. Badgwell, and R. B. Hawkins, "Robust steady-state target calculation for model predictive control," AIChE Journal, vol. 46, no. 5, pp. 1007-1024, 2000.

[22] Y. G. Xi and H. Y. Gu, "Feasibility analysis of constrained multi-objective multi-degree-of-freedom optimization control in industrial processes," Acta Automatica Sinica, vol. 24, pp. 727732,1998

[23] T. Zou, H. Q. Li, X. X. Zhang, Y. Gu, and H. Y. Su, "Feasibility and soft constraint of steady state target calculation layer in LPMPC and QP-MPC cascade control systems," in Proceedings of the International Symposium on Advanced Control of Industrial Processes (ADCONIP '11), pp. 524-529, May 2011.

[24] Y. G. Xi, Predictive Control, National Defense Industry Press, Beijing, China, 1993. 


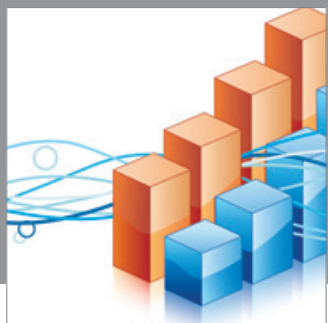

Advances in

Operations Research

mansans

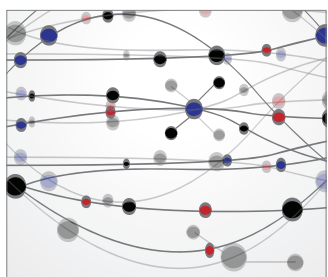

The Scientific World Journal
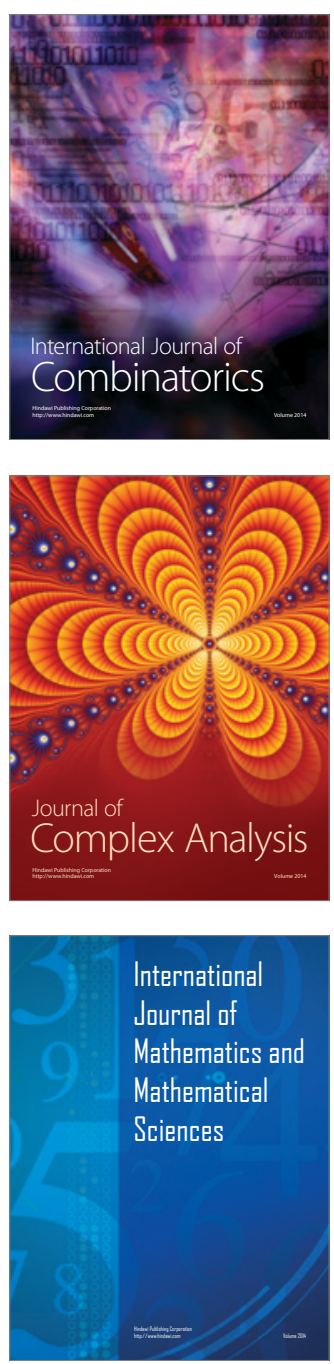
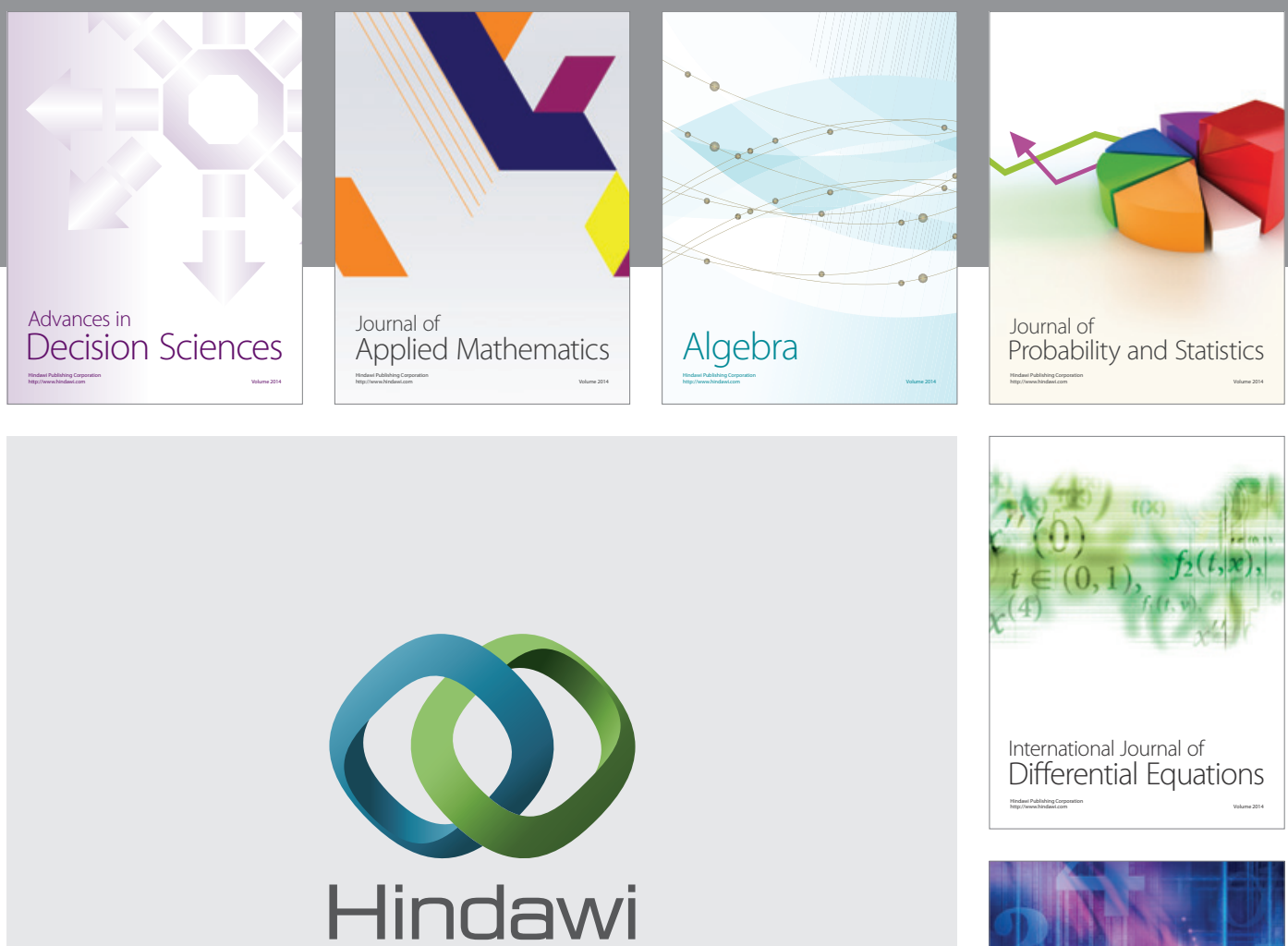

Submit your manuscripts at http://www.hindawi.com
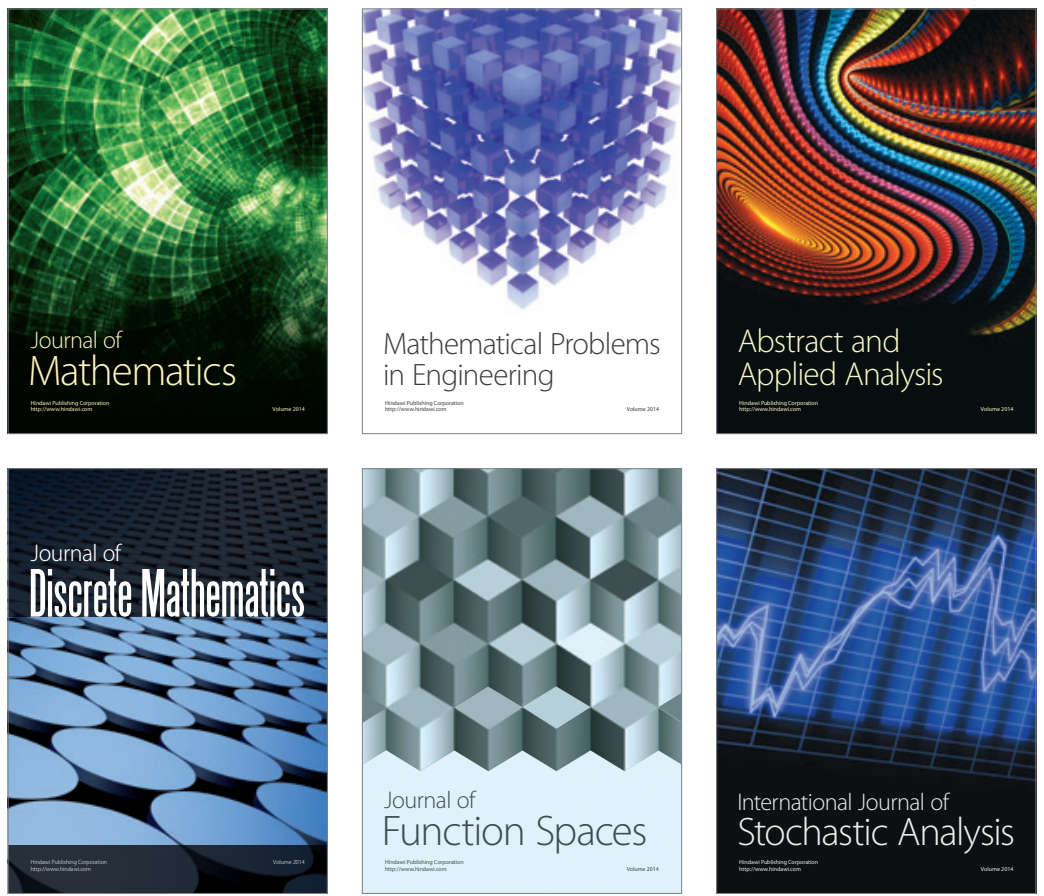

Journal of

Function Spaces

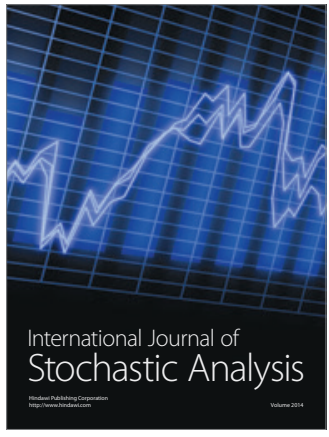

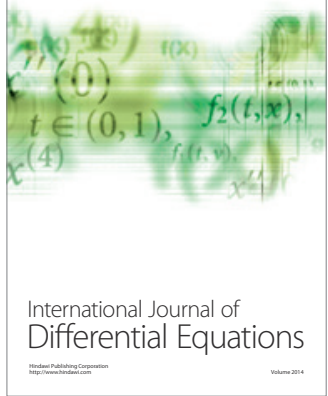
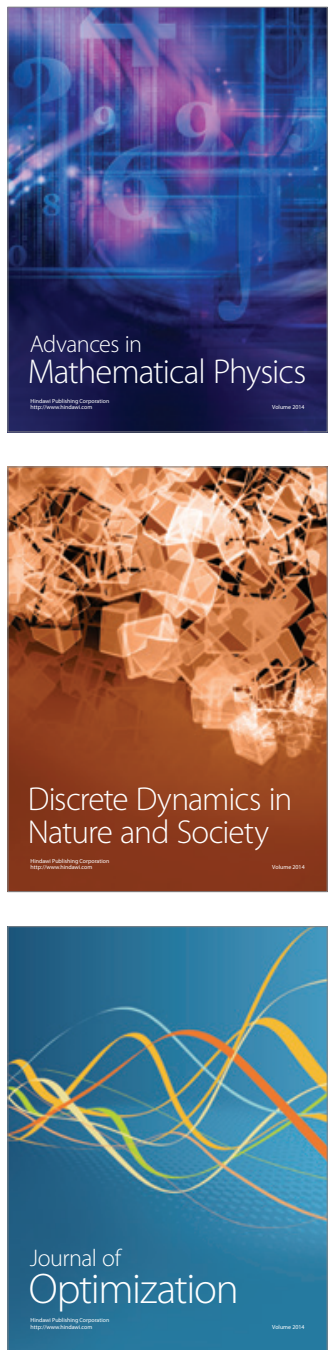